\title{
Integrated Methodology for the Design of Formulated Products Incorporating Consumer Preferences and Heuristic Knowledge
}

\author{
Javier Andrés Arrieta Escobar ${ }^{1}$, Mauricio Camargo $^{2},{\text { Laure } \mathrm{Morel}^{3} \text {, Fernando Bernardo }}^{4}$, \\ Alvaro Orjuela ${ }^{5}$, and Laurent Wendling 6 \\ ${ }^{1}$ Universite de Lorraine \\ ${ }^{2}$ Université de Lorraine Equipe de Recherche des Processus Innovatifs, ERPI-ENSGSI \\ ${ }^{3}$ Université de Lorraine \\ ${ }^{4}$ University of Coimbra \\ ${ }^{5}$ Universidad Nacional de Colombia \\ ${ }^{6}$ Paris Descartes University University Institute of Technology Paris Descartes
}

May 6, 2020

\begin{abstract}
This work proposes a systematic approach for the design of formulated products incorporating consumer assessment and heuristic knowledge. The methodology is divided into two main phases: Consumer assessment and product realization. In the first part, product attributes valued by consumers and their interactions are identified through usability tests performed on a reference product, and fuzzy integral analysis. In the second phase, alternative product formulations are generated using a mixed-integer optimization approach, which incorporates heuristic rules regarding the choice of ingredients and their amounts. The results from the consumer assessment phase are transformed into additional heuristics and incorporated into the design. Using two commercial skin moisturizers as references, different alternative formulations are generated at a lab scale. Those formulations that included additional heuristics are shown to be more similar to the references. As a result, the incorporation of consumer preferences significantly reduced the time and resources spent on the design process.
\end{abstract}

\section{Introduction}

The design of optimal mixtures is considered an important challenge in many industries, especially for the manufacturing of formulated consumer products. Due to the infinite number of possible combinations of ingredients and their corresponding concentrations, a critical issue is how to define a reduced search space during early stages to accelerate the design process, using the available knowledge. Besides the key physicochemical properties of the final product, it is of paramount importance to account for the product performance as perceived by the final consumer. The fulfillment of this goal requires greater involvement of consumer needs into the product design inputs.

Typically, the design process of formulated consumer products corresponds to an iterative succession of 4 steps, as shown in Figure 1. ${ }^{1,2}$ Within this process, the marketing team is normally responsible for the first two steps (1 and 2) concerning the consumer assessment phase, where the information on the user needs is translated into basic functional specifications of the product. ${ }^{1,3}$ 


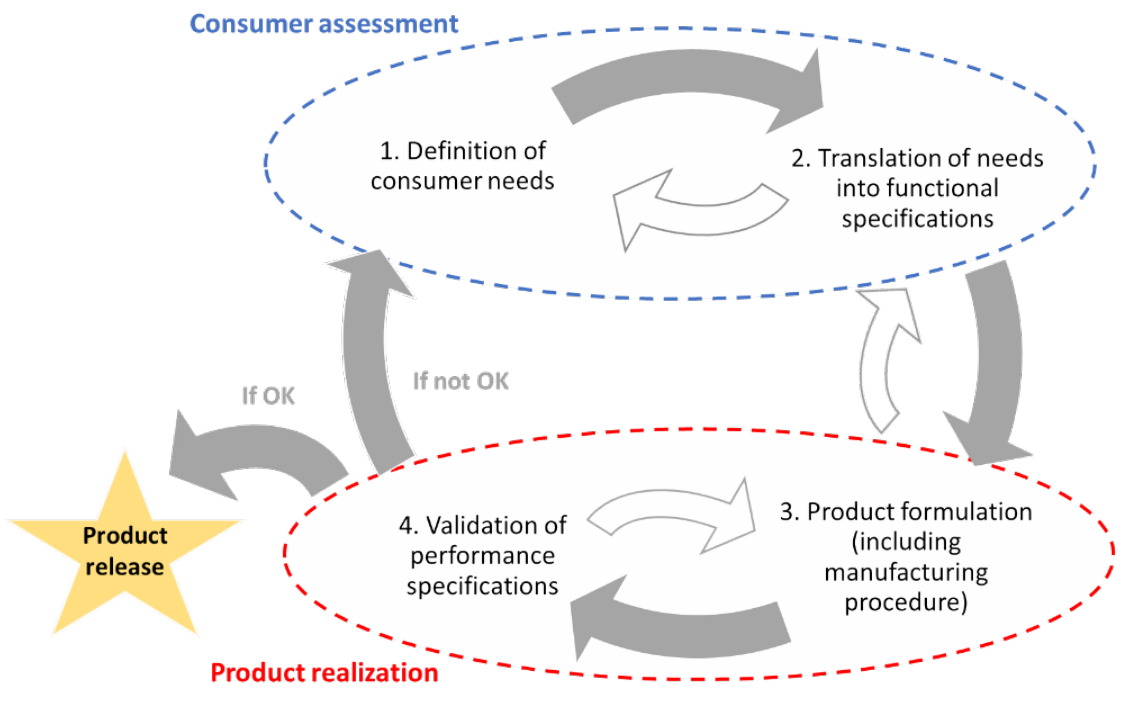

Figure 1: Formulated consumer product design cycle. Adapted from the original pictures ${ }^{1,2}$

Then, in the product realization phase, technical teams are in charge of the next steps (steps 3 and 4 in Figure 1): product formulation, prototyping, manufacturing, and validation of required specifications. At the end of this cycle, if the prototype meets the given specifications, the product is ready to be launched into the market; otherwise, the process should restart. Very often, modifications are made in terms of the composition, along with the product realization phase. Their consequences in product performance may be multiple and hard to predict. Product reformulations may significantly affect product properties, including changes in product microstructure and thus in the perception of the product by the consumer. ${ }^{4}$

An important feature of formulated consumer products is that users generally do not assess their value based on technical specifications, but rather according to functionality and performance attributes, which are often referred to as the quality factors. ${ }^{5}$ Because quality factors are sometimes qualitative and/or subjective, performance metrics need to be established. To improve the traditional trial-and-error procedure during this phase, many authors have developed a knowledge basis for the collection of the consumer needs, their translation into necessary categories of ingredients, and the desired end-use properties, particularly in the case of cosmetics and personal care products. ${ }^{6-13}$ In the context of emulsions, Mattei ${ }^{13}$ proposed such a knowledge basis, including consumer needs, categories of ingredients, and end-use properties with target values and boundaries of acceptance. However, one of the main challenges still to be solved is related to the formalization of the consumer needs and their translation into physicochemical properties, namely for product attributes of which assessment is inherently subjective, such as those related to human senses. ${ }^{14}$ This aspect is particularly relevant for cosmetics, as there is an important influence of the sensory interaction on the users' perception and assessment of the product. ${ }^{15}$ To measure this, normally, an objective evaluation (i.e. sensorial profiling) is made by a panel of experts under defined conditions, ${ }^{16}$ which makes it a very resource-intensive procedure. For this reason, researchers have investigated the characterization of sensory product attributes, mainly translating them to textural and rheological properties. ${ }^{17-19}$ Nevertheless, these tests are performed by evaluating a single dimension, whereas cosmetic performance evaluation is the result of a combination of sensory, emotional and rational responses during the experience of using the product. ${ }^{20}$

Since cosmetics or food products are conceptually designed to match consumer expectations, it is important to access the implicit emotions people have when interacting with the products and translate them into product specifications. ${ }^{21,22}$ "Affective Engineering" or "Kansei Engineering" approaches can be used to study how pleasure and efficiency are linked together during the experience of using a product. ${ }^{23,24}$ In such approaches, semantic attributes (SA) have been used to describe the affective responses of consumers to products. ${ }^{25}$ 
Traditional techniques, such as PCA (Principal Components Analysis) or multi-factorial analysis, are useful to reduce the semantic space and to quantify the relative importance of each SA or the correlation among them. However, these tools are not suitable to quantify the interactions (i.e. the synergistic or antagonist effects) among groups of SA. For this task, multicriteria techniques, such as the Multiple-Utility Attribute Theory (MAUT) or Analytic Hierarchy Process (AHP) could be used. ${ }^{26}$ Nevertheless, dealing simultaneously with the set of multidimensional semantic attributes and their interaction is not a trivial task.

Fuzzy measures have been applied to the subjective evaluation of different attributes, such as color or texture. ${ }^{27,28}$ Recently, a methodology to integrate the user's perception and identify the importance and interaction of consumer attributes, based on fuzzy measures, was proposed by Camargo et al in the context of massing soles. ${ }^{29}$ On the other hand, we had already proposed a systematic method to generate and select alternative formulations, taking into account both available quantitative property models as well as heuristic formulation rules, with these last one being translated into algebraic restrictions, thus providing a reduced set of alternatives to be prototyped. ${ }^{30,31}$ In this paper, we combine these two complementary approaches, using the methodology of consumer assessment to understand the importance level of each quality factor along with their synergies or antagonisms ${ }^{29}$ and then integrate this knowledge into the product realization phase, based on our method of computer-aided generation of alternative plausible formulations. ${ }^{31}$ This methodology was partially presented in a short paper, ${ }^{32}$ being now fully discussed and illustrated, including two examples of skin moisturizers design.

\section{An integrated methodology for formulated products design}

As schematically presented in Figure 2, the proposed integrated methodology consists of two main phases: consumer assessment and product realization. In the first one, consumer preferences are assessed, and the outputs converted into a set of heuristics. These are then integrated into the second phase of product realization, where product modeling is carried out based on available quantitative property models and heuristic rules converted into algebraic restrictions.

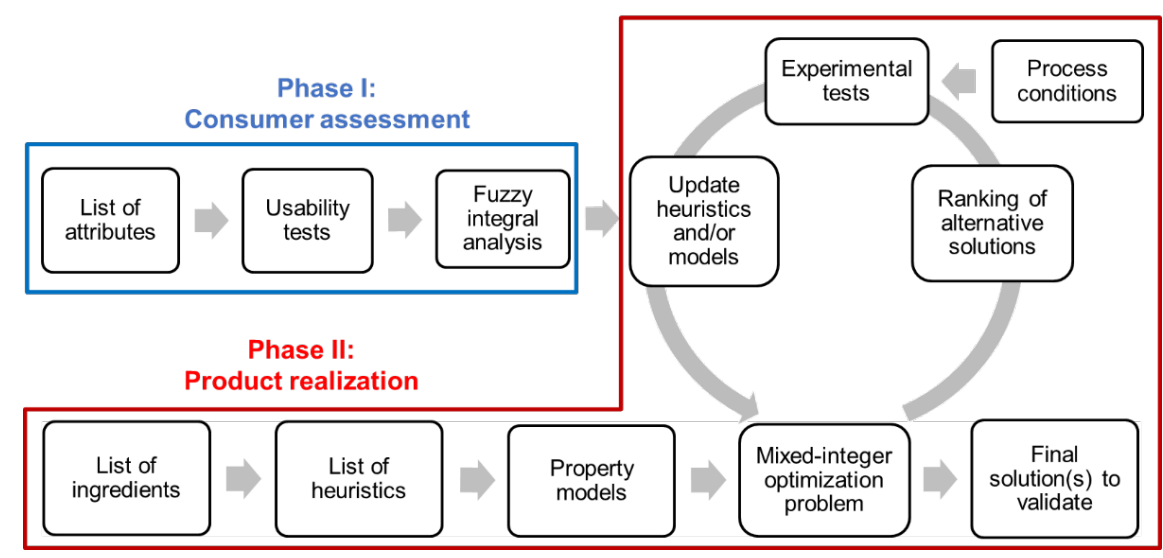

Figure 2: An integrated methodological approach for the design of formulated products

\section{Phase I: Consumer assessment}

In this phase, the methodology proposed by Camargo et al. ${ }^{29}$ was adapted to identify and qualify, in terms of importance and interaction among them, the set of semantic attributes that best describes the feelings of a group of users and then to calculate the fuzzy measures. This method employs the backpropagation algorithm with constraints proposed by Grabisch ${ }^{33,34}$ to approximate the fuzzy measure accurately, using the Choquet integral. ${ }^{35}$ This algorithm is efficient when training data are limited, has a low computing time, and a low memory cost. ${ }^{28}$ As the input range is not strictly defined in this study, learning is combined here with a linear output based on linear error regression to ensure an accurate decision and a better convergence. ${ }^{36}$ 
The details of the definitions and computations of Choquet integral and fuzzy measures are presented in the Section A of the Supplementary material.

List of attributes : As for most formulated consumer products, the definition of product attributes for cosmetics constitutes the main input required and is usually obtained from multiple sources, such as market and customer surveys, patent, literature, among others. ${ }^{37}$ Once the set of words defining product attributes is established (i.e. SA), the next step is a reduction in the number of words by excluding irrelevant or repeated words. We used experts opinions to this purpose, but several other techniques, such as affinity diagrams, factor analysis or Principal Component Analysis (PCA), may also be applied. ${ }^{15,29}$

Usability tests : A questionnaire is prepared to evaluate the entire set of identified SA. Participants in the test should be regular users of the target product, as they can perceive differences even if they have no common references of the attributes' scales. ${ }^{38}$ The participants of the test are asked to rate a selected prototype on a five-level ordinal scale, using a semantic differential method. ${ }^{39}$ A typical prototype could be the most sold product for the chosen category or any other product established as a reference. It is important to underline that a learning output, represented for example by global acceptability or a purchase intention, should be included in the evaluation so the fuzzy measures can be calculated. ${ }^{29}$

Fuzzy integral analysis: The main equations to calculate the fuzzy measures $(\mu)$ are shown in the section A of the Supplementary Material. Once the fuzzy measures for each SA ( $i$ in a set of $n$ ) are calculated, the importance and interaction indices could be estimated using the Shapley and the Murofushi and Soneda indices, respectively. The importance index $(\sigma)$ is based on the definition proposed by Shapley in the game theory: ${ }^{40}$

$\sigma(\mu, i)=\frac{1}{n} \sum_{t=0}^{n=1} \frac{1}{\left(\frac{n-1}{t}\right)_{|T|=i}} \sum_{T \subseteq X i}[\mu(T \cup i)-\mu(T)](1)$

Once normalized, the Shapley index can be interpreted as a weighted average value of the marginal contribution of each criterion in all combinations, so the sum of the index of all SA is equal to $1 .{ }^{45}$

On the other hand, the Murofushi and Soneda indices $(I)$ represent the degree of interaction between two $\mathrm{SA}(i, j \ldots n):^{41}$

$I(\mu, i j)=\sum_{T \subseteq \mathrm{Xij}_{\mathrm{ij}}} \frac{(n-t-2) ! t !}{n-1}[\mu(T \cup i j)+\mu(T)-\mu(T \cup i)-\mu(T \cup j)](2)$

These indices and their interactions (positive or negative) could be used to validate the relevance of the selected SA. A positive interaction index for two SA means that the importance of one SA is reinforced by the second. A negative interaction index indicates that the SAs are antagonists, and their combined use impairs the final decision. From these two indices, a third one called composite index can be calculated as a normalized product of them, and it serves to guide the selection of the alternative formulations in the next phase.

\section{Phase II: Product realization}

This part of the methodology starts by identifying a list of available ingredients. For instance, cosmetic product designers are constrained to use approved ingredients, or they are limited to choose among available ingredients in the company's inventory. Here, a binary variable is associated with each potential ingredient, indicating whether it is selected or not as part of the formulation. Then, available heuristics regarding the choice of ingredients and their amounts are listed and modeled as algebraic restrictions. Some heuristics are first stated as logical conditions and then translated into algebraic constraints involving binary variables. These heuristic-related restrictions, together with other known limits (technical and/or legal), help to reduce the design space. The search in this reduced space is then guided by available property models relating product composition to key physicochemical properties or even to sensorial attributes.

Let $y$ be the vector of binary variables associated with the choice of ingredients and $x$ the vector of corresponding their concentration in the final product (i.e. mass fractions). Let $p$ be the vector of product 
performance metrics, including well-defined physicochemical properties and metrics related to more subjective sensorial attributes. Product quality is often evaluated in terms of the deviation of $p$ from target values $p^{*}$ (product performance specifications). Then, property models, also known as the property function, are any relationship between metrics $p$ and product composition, here represented by the set of equations $h_{(x, y, p)}=0$ .${ }^{42}$ The heuristic rules are incorporated in the problem formulation as the set of constraints $g_{(x, y, p)[?]} 0$. Finally, let $f$ be a global objective function to be minimized, in this case accounting for both product quality deviation and costs. The problem of optimal product formulation may then be stated as follows:

\begin{tabular}{lll}
\hline$f\left(x, y, p, p^{*}\right) \quad$ [product performance] & $f\left(x, y, p, p^{*}\right)$ [product performance] \\
s.t. & $h_{1}(x, y, p)=0$ [property function] \\
& $g_{1}(x, y, p) \leq 0$ [heuristic-related restrictions] \\
& $g_{2}(x, y, p) \leq 0$ [other restrictions] \\
\hline
\end{tabular}

As sketched in Phase II of Figure 2 above, computer-generated alternatives and their realization should evolve in successive cycles where results from experimental tests are fed back to problem formulation (3), in the form of updated models and heuristics. When several alternative formulations are required, a rank of feasible solutions with increasing values of the objective $f$ may be generated through integer cuts. ${ }^{30}$ In any case, successive cycles should desirably result in an optimal alternative, which meets all product specifications and integrate the consumer assessment. Most heuristic rules have a linear formulation, however, property and process functions are likely to be non-linear, so problems in the form of (3) will be MINLP problems, as it is illustrated in the case study.

In summary, a general methodological approach is presented here for the design of formulated products, in which a fuzzy measure analysis for the consumer assessment, along with heuristics and property models incorporation for the product realization phase, are used to guide the selection of formulations that solve the original design problem, using the reformulations of cosmetic emulsions as case studies.

\section{Cosmetic emulsions case study}

The proposed methodology was tested in the design of cosmetic products, specifically skin moisturizers. These products are used to keep in good condition the skin by maintaining its balance of oil and water. ${ }^{43}$ Skin moisturizers are generally oil in water $(\mathrm{O} / \mathrm{W})$ emulsions, and commercially they are usually divided into two main types: creams and lotions. Lotions are low-viscosity emulsions, while creams are much higher viscosity materials, generally presented as semi-solid emulsions. ${ }^{44}$ The $\mathrm{O} / \mathrm{W}$ emulsions are characterized by a low internal phase ratio, typically containing 10 to $35 \%$ dispersed phase. ${ }^{45}$ For this reason, the addition of a suitable emulsifier agent along with the application of mechanical agitation is necessary to create a stable emulsion. ${ }^{46}$ In this work, apart from some mandatory ingredients like water or humectants (i.e. Glycerol) that are kept constant, three main types of ingredients are considered for the modeling of $\mathrm{O} / \mathrm{W}$ emulsions: Emollients in the dispersed phase, thickeners in the continuous phase, and emulsifiers in the interface. The selection of these groups of ingredients is based on several studies that evaluated their impact on the sensorial, rheological and textural properties of cosmetic emulsions. ${ }^{19,46-50}$

When formulating cosmetic emulsions, the dispersed phase should be first selected, ${ }^{3}$ and emollients constitute the main component of this phase, particularly in $\mathrm{O} / \mathrm{W}$ emulsions. Emollients are required in the dispersed phase because they help to prevent soaping, they improve spreadability, and they are responsible for the consumer-perceived benefits after evaporation of volatile materials. ${ }^{46,48}$ Performance of emollients is generally related to greasiness, and the emulsifying properties are dependent on other physical properties such as density, viscosity, melting point, and the required HLB (RHLB). ${ }^{51,52}$

Emulsifiers are essential ingredients to stabilize the emulsions. ${ }^{45}$ The type of surfactant and its physicochemical properties will influence the droplet size and stability of the emulsion. ${ }^{52}$ HLB is a used here to predict the emulsifying properties of surfactants ${ }^{37}$ and to correlate with some sensorial properties of the emulsions, such as color, odor, and consistency. ${ }^{46,53}$ 
Thickeners are used to increase the viscosity of the continuous phase and to mitigate the phase separation. ${ }^{46,51}$ It has also been shown that thickeners could have a relevant impact on skin feeling, namely when removing cream from the container, and when spreading the product. ${ }^{54}$

Phase I: Consumer assessment

List of attributes : In the first step of the consumer assessment phase, eight attributes reported in the literature were chosen to be evaluated during the usability tests. ${ }^{8,55}$ Each set of words describing the attributes was selected after asking four experts to brainstorm keywords that could be used to describe skin moisturizers, and then to classify them in each category. The two most repeated terms were selected to represent each attribute, as shown in Table 1.

Table 1. List of main attributes for cosmetic emulsions

\begin{tabular}{lll}
\hline Attribute & Word 1 & Word 2 \\
Thickness & Thick & Thin* \\
Spreadability & Easy to spread & Glides easily \\
Stickiness & Sticky & Light* \\
Ease of absorption & Easily absorbed & Penetrates quickly \\
Freshness & Fresh sensation & Cold sensation \\
Residues & Whitening on rub-out & Leaves residues \\
Greasiness/oiliness & Greasy & Oily sensation \\
Moisturization & More hydrated skin & Softer skin \\
\hline
\end{tabular}

*Pair of words are at opposite extremes of the attribute scale

Usability tests and fuzzy integral analysis : To define consumer needs regarding the use of a cosmetic moisturizer, usability tests were conducted during two main popular events in the city of Nancy (France). Two groups, consisting of thirty-two female (average age 31 years old), tested one of the two selected reference products: a cream - Sample 1 (Atoderm@ Ultra-nourishing Cream, Bioderma), and a lotion - Sample 2 (NIVEA $\AA$ Body Sun protection FPS 15, Beiersdorf). and answered the set of questions corresponding to each word in Table 1, using a 5-level ordinal scale (i.e. from not at all to very much). The complete survey form - in French (original) and English - and the results are presented in the Section B of the Supplementary material.

Phase II: Product realization

Given a defined list of ingredients, the formulation of the cosmetic emulsions should be conducted according to the procedure described in Figure 2.

List of ingredients, heuristics, and property models:

Emollients: A proper combination of three or more emollients of a high, medium, and low spreading types provides the complete profile for a well-performing product. ${ }^{56}$ The residual film of the emollient helps to lubricate and reduce the friction exerted on the skin surface. ${ }^{57}$ The greasiness value $(\gamma)$ of a mixture of emollients was estimated here as a weighted average from individual values. ${ }^{58}$ Typical greasiness values for skin moisturizers are in the range from 2.0 and 2.4 on a five-point scale. ${ }^{8}$ The RHLB value of the emollients was obtained directly from the ingredient provider or other sources. ${ }^{1}$ The list of the emollients, grouped by its spreading properties, is available in Table C1 of the Supplementary Material.

Emulsifiers: Generally, a suitable combination of at least two nonionic surfactants should be used at a minimum level of $2 \%$ and up to $5 \%$ in a skin moisturizer. ${ }^{6,59}$ The final HLB value of the combination should be between 8 and 15 to assure a stable $\mathrm{O} / \mathrm{W}$ emulsion. This is generally achieved by combining one part of a medium HLB (9-15) emulsifier, three to six parts of a high HLB emulsifier ( $>16)$, and two to six parts of a low HLB emulsifier $(<8)$. Also, a total surfactant/oil ratio of 1:4 to 1:6 should be used to stabilize the 
resulting emulsion. ${ }^{60}$ The classical empirical HLB model is here adopted, namely, the HLB of the surfactant mixture should approximately match the HLB required by the oil mixture: HLB = RHLB. The list of the emulsifiers used in this study is available in Table C-2 in the Supplementary Material.

Thickeners: Thickeners commonly used in skin moisturizers are water-dispersible polymers and fatty alcohols. Normally, they are used simultaneously in skincare emulsions, as these formulations are more prone to destabilization. ${ }^{60}$ The list of the thickeners used in this study is on Table C-3 in the Supplementary Material.

Thickeners influence the product's perception, particularly what is called the primary and secondary skin feelings. ${ }^{54,61}$ The primary feeling is correlated to the viscosity perceived at the onset of the flow of the product $\left(\eta_{1}\right.$, viscosity at low shear stresses), while the secondary feeling corresponds to a much lower final viscosity, perceived during the product's application ( $\eta_{2}$, viscosity at high shear stresses). Under such circumstances, the ideal value of $\eta_{1}$ is between 1350 and $3500 \mathrm{~Pa} \cdot \mathrm{s}$ for creams and between 120 and $500 \mathrm{~Pa} \cdot \mathrm{s}$ for lotions. In contrast, $\eta_{2}$ should be between 0.023 and $0.500 \mathrm{~Pa} \cdot \mathrm{s}$ for both types of product. This last viscosity interval corresponds to a shear rate of around $500 \mathrm{~s}^{-1}$ if the application is over small areas (e.g. face), or $5000 \mathrm{~s}^{-1}$ if the application is over large areas of the body. ${ }^{54}$

Composition-viscosity data for polymer thickened aqueous solutions, ${ }^{62}$ together with reliable theoretical models to predict the effect of the dispersed phase, ${ }^{63}$ were used to construct equations of the type $\log (\eta)=$ $a+b x_{n}+c \phi$ (average relative error of $22 \%$ ), for each polymer, and for both $\eta_{1}$ and $\eta_{2}$ ( $\phi$ is the mass fraction of oil phase). Since only one polymer is used, mixing rules are not needed. Fatty alcohols were used at a $2-4 \%$ concentration. As indicated above, all the formulations also included a fixed amount of mandatory ingredients, namely water, humectant (3\% Glycerol) and preservative ( $0.7 \%$ Cosgard@) .

Optimization problem : Product design variables and short formulation of the optimization problem for both case studies - in the form of (3) - are shown in Table 2.

Table 2. Product design variables and initial problem formulation

Binary variables $y$ (choice of ingredients) and continuous variables $x$ (wt \%)

high spreading emollient: $y_{i}, x_{i}$ medium spreading emollient: $y_{j}, x_{j}$ low spreading emollient: $y_{k}, x_{k}$ thickening polymer: $y$

Emollients, nonionic surfactants, and thickeners (polymers or fatty alcohols) are selected from an initial list with a total of 36 ingredients, as presented in Tables C-1 to 3 in Supplementary Material. Additional restrictions were added to this problem formulation when integrating the consumer assessment in each case. Other heuristics are transformed into variables and correlated in equations employing propositional logic, as described in a previous contribution from the authors. ${ }^{30}$ Vectors of decision variables - binary $(y)$ and continuous $(x)$ - both have a dimension of 36 . The chosen objective function was defined as the total cost of the formulation (USD $/ \mathrm{kg}$ ), only considering the unit cost of each ingredient and excluding fixed ingredients. Manufacture costs were not considered.

The restrictions involving RHLB and HLB are the only source of non-linearity.

$$
\begin{aligned}
& R H L B=\frac{\sum x_{i} \bullet \mathrm{RHLB}_{i}+\sum x_{j} \bullet \mathrm{RHLB}_{j}+\sum x_{k} \bullet \mathrm{RHLB}_{k}}{\sum y_{i} \bullet x_{i}+\sum y_{j} \bullet x_{j}+\sum y_{k} \bullet x_{k}} ; H L B=\frac{\sum x_{r} \bullet \mathrm{HLB}_{r}}{\sum y_{r} \bullet x_{r}}(4) \\
& \left(\sum x_{i} \bullet \mathrm{RHLB}_{i}+\sum x_{j} \bullet \mathrm{RHLB}_{j}+\sum x_{k} \bullet \mathrm{RHLB}_{k}\right) \bullet\left(\sum y_{r} \bullet x_{r}\right)-\left(\sum y_{i} \bullet x_{i}+\sum y_{j} \bullet x_{j}+\sum y_{k} \bullet x_{k}\right) \bullet \\
& \left(\sum x_{r} \bullet \mathrm{HLB}_{r}\right)=0 \quad(5)
\end{aligned}
$$

The resulting MINLP problem can be solved in less than one second using the GAMS platform and the global solver BARON. The optimal solution is selected in each case to be compared with the commercial sample.

Experimental tests and emulsions characterization: The complete list of chemicals used in the prototyping 
of the alternative emulsions is presented in the Table C-4 of the Supplementary Material. They were used as obtained without further treatment.

Rheology and textural analysis: The rheological analysis was performed on a rotational viscometer ARES (TA Instruments, New Castle, USA). The flow behavior was studied by continuous shear investigations, which were performed to evaluate the shear response $(\mathrm{Pa} \cdot \mathrm{s})$ as a function of shear rate $\left(\mathrm{s}^{-1}\right)$ (from 0.01 to around $500 \mathrm{~s}^{-1}$ ) with a logarithmically increasing scale (5 points), using parallel plates $(25 \mathrm{~mm})$. All samples were tested one week after preparation to assure that the emulsions were stable. The gap between the disc and the plate $(0.5 \mathrm{~mm})$ was carefully filled with a sample of the product, and any left material was removed using a metal spatula. All measurements were conducted at $20+-0.1 \operatorname{deg} \mathrm{C}$ and at least in duplicate.

The textural properties of the skin moisturizer samples were measured using a Texture Analyzer TA.XT Plus (Stable Micro Systems Ltd., Godalming, U.K.). The unit had a load cell of $30 \mathrm{~kg}$, with an A/BE back extrusion rig, which included a locating base plate, sample containers (50mm internal diameter), and compression discs (35mm diameter). After penetrating the sample $(25 \mathrm{~mm}$ deep) at a rate of $2.0 \mathrm{~mm} / \mathrm{s}$, the probe returned to the initial position. Following this procedure, the firmness, consistency (related to hardness), cohesiveness, and index of viscosity (related to adhesiveness) of the samples were measured in duplicate and average values were calculated for all parameters (all expressed as positive values). All the measurements were taken at room temperature of $20+-0.2 \mathrm{deg}$.

Microscopy: Emulsions were observed using a portable microscope Dino-Lite(r) Edge AM7515MT8A (Taipei, Taiwan), with a magnification of 900x, and images were captured with the in-built camera 5Mpixels (2592x1944). For this, a small amount of sample was mounted on a glass slide along with a drop of distilled water under a coverslip. All samples were examined two months after preparation to assure long term stability.

Evaluation of white residues after product application on skin:An adaptation to the descriptive skin-feel analysis method described by ASTM E1490-0 $3^{64}$ was implemented here to establish a qualitative comparison of the white residues left by two products during rub-out. In this case, $0.1 \mathrm{~g}$ of each product was spread on the mid-section of the forearm, using the index finger for one product and the middle finger for the other product, both from the opposite hand. The white residues of both samples were compared after 15 circular rubs at a rate of two strokes per second.

Integration: Heuristics update and validation

This phase was carried out to do the screening of the prototype products, and to iterate within the design process to enhance product performance, as part of the cyclic part in Figure 2. This is done by integrating consumer assessment in the product realization phase. Depending on the experimental tests, the ranking of attributes, and their interactions, a series of heuristics could be transformed into additional restrictions in the algorithm, as shown in Table 3.

Table 3. Heuristics to guide the selection of alternatives based on selected attributes.

Attribute

Thickness

White residues after rub-out

Greasiness /oiliness

\section{Actions to increase}

Adjust viscosity target value in the algorithm Increase fatty alcohol content 60
Actions to reduce
Adjust viscosity target value in
the algorithm Replace part of
surfactants to those having lauryl
or oleyl alkyl group Add
branched-chain higher alcohol (i.e.
Octyldodecanol) 60
Add up to $2 \%$ low HLB emulsifier
and allow HLB $<$ RHLB Add
silicones to the formulation 65
Adjust greasiness target value in
the algorithm 
These actions are taken to increase or reduce the value of a specific attribute, depending on the case. For instance, the presence of white residues after rubbing is mainly related to the generation of microfoam (emulsifiers "catching" air), so the use of silicones could also reduce this problem. Another possible solution is to add up to a $2 \%$ low HLB emulsifier and allow the HLB to be lower than the RHLB. This will not only remove the microfoam but also increase the viscosity of the formulation. In this case, (5) will change to:

$0.5 \leq\left(\sum y_{i} \bullet x_{i}+\sum y_{j} \bullet x_{j}+\sum y_{k} \bullet x_{k}\right) \bullet\left(\sum x_{r} \bullet \mathrm{HLB}_{r}\right)-\left(\sum x_{i} \bullet \mathrm{RHLB}_{i}+\sum x_{j} \bullet \mathrm{RHLB}_{j}+\sum x_{k} \bullet \mathrm{RHLB}_{k}\right) \bullet$ $\left(\sum y_{r} \bullet x_{r}\right) \leq 1(6)$

This example emphasizes the importance of heuristics updating, and the set of equations required for properties prediction in the new design iteration.

\section{Results and discussion}

Phase I: Consumer assessment

Table 4 contains the resulting values of each assessed attribute during the consumers' evaluation. The participants were asked to rate the different attributes on a 5-level ordinal scale and then the values were normalized (0 to 1 scale).

Table 4 : Mean and variance of the scores for the attributes with corresponding Shapley indices.

\begin{tabular}{llllll}
\hline Attribute & Symbol & $\begin{array}{l}\text { Sample 1 - Cream } \\
\text { Mean }\end{array}$ & $\begin{array}{l}\text { Sample 1 - Cream } \\
\text { Variance }\end{array}$ & $\begin{array}{l}\text { Sample 1 - Cream } \\
\text { Shapley index }\end{array}$ & $\begin{array}{l}\text { Sample 2 - L } \\
\text { Mean }\end{array}$ \\
Thickness $^{\text {a,b }}$ & $\mathrm{V}$ & 0.46 & 0.059 & 1.05 & 0.26 \\
Spreadability $^{\mathrm{a}, \mathrm{b}}$ & $\mathrm{S}$ & 0.75 & 0.028 & 0.35 & 0.84 \\
Stickiness $^{\mathrm{a}, \mathrm{b}}$ & $\mathrm{P}$ & 0.45 & 0.036 & 1.19 & 0.72 \\
Ease of absorption $^{\mathrm{a}, \mathrm{b}}$ & $\mathrm{A}$ & 0.35 & 0.055 & $\mathbf{1 . 5 7}$ & 0.61 \\
Freshness $^{\mathrm{a}, \mathrm{b}}$ & $\mathrm{F}$ & 0.31 & 0.040 & $\mathbf{1 . 6 5}$ & 0.45 \\
Residues $^{\mathrm{b}}$ & $\mathrm{R}$ & 0.30 & 0.043 & $\mathbf{1 . 2 2}$ & 0.24 \\
Greasiness/oiliness $^{\mathrm{a}, \mathrm{b}}$ & $\mathrm{G}$ & 0.74 & 0.040 & 0.38 & 0.46 \\
Moisturization $^{\mathrm{a}, \mathrm{b}}$ & $\mathrm{H}$ & 0.61 & 0.039 & 0.59 & 0.73 \\
Overall performance $^{\mathrm{a}, \mathrm{b}}$ & $\mathrm{C}$ & 0.43 & 0.074 & & 0.57 \\
\hline
\end{tabular}

${ }^{\mathrm{a}}$ Different mean, t-test $(\mathrm{p}<0.05){ }^{\mathrm{b}}$ Same variance, F-test $(\mathrm{p}>0.05)$

Collected data were verified to evaluate its consistency, and a correlation with the normal distribution above 0.92 was obtained for all attributes, as presented in Section D of the Supplementary material. According to results, the mean score for each attribute was different between the two samples, except for the "residues" score. Here it is important to mention that both samples had low values in the "residues" attribute score, which means that the emulsions left almost no residue or whitening after spreading and rubbing.

The corresponding Shapley indices (1) for each attribute computed from the fuzzy integral analysis are also presented in Table 4. The three higher values for each sample are marked in bold characters. From these results, it is possible to see that "freshness" is the most important attribute for Sample 1 (1.65), followed by "ease of absorption" (1.57) and "residues" (1.22). The first two attributes were poorly graded in the test (0.31 and 0.35 in Table 4, respectively), which suggests that the heavy emollience of the product could influence the overall score. Indeed, Sample 1 contains mineral oil, an occlusive ingredient that hinders water evaporation from the skin, which is usually related to a low freshness sensation. ${ }^{55}$ In the case of Sample 2 , "residues" has the highest index (i.e. 1.74) followed by "thickness" (i.e. 1.36) and "freshness" (i.e. 1.26). Among these attributes, "thickness" is also the lowest rated (0.26 in Table 4), which could indicate that Sample 2 is perceived as not being thick enough, thus affecting the overall performance score of the product. Similar results have been reported by other authors when the product has a low viscosity. ${ }^{66}$ 
Murofushi and Soneda or interaction indices (2) are listed in Table 5. The three highest-values for each pair of attributes and the total interactions alike are marked in bold characters. It can be observed that the attribute "residues" is consistently interacting with other attributes and appears amongst the highest ranked attributes for total interactions in both cases (4.00 for Sample 1 and 4.02 for Sample 2). This attribute has also the highest values of mutual interactions, namely with "ease of absorption" (1.46) and "freshness" (1.15) in the case of sample 1, and with "thickness" (0.69), "stickiness" (0.97) and "moisturization" (-0.85) in the case of sample 2. The positive or negative sign means that the effect is synergistic or antagonist, viscosity. ${ }^{66}$

Table 5. Murofushi and Soneda indices. Symbols according to Table 4

\begin{tabular}{|c|c|c|c|c|c|c|c|c|c|}
\hline & & V & $\mathbf{S}$ & $\mathbf{P}$ & $\mathbf{A}$ & $\mathbf{F}$ & $\mathbf{R}$ & G & $\mathbf{H}$ \\
\hline \multirow[t]{8}{*}{$\begin{array}{l}\text { Sample } \\
1 \text { - } \\
\text { Cream }\end{array}$} & $\mathbf{V}$ & - & 0.31 & -0.30 & 0.10 & 0.13 & 0.51 & 0.10 & 0.15 \\
\hline & $\mathbf{S}$ & & - & 0.31 & 0.28 & 0.11 & 0.21 & -0.14 & -0.10 \\
\hline & $\mathbf{P}$ & & & - & 0.41 & -0.29 & 0.63 & 0.01 & 0.21 \\
\hline & $\mathbf{A}$ & & & & - & 1.15 & 1.46 & 0.81 & -0.22 \\
\hline & $\mathbf{F}$ & & & & & - & 0.98 & 0.06 & 0.85 \\
\hline & $\mathbf{R}$ & & & & & & - & 0.11 & 0.08 \\
\hline & $\mathbf{G}$ & & & & & & & - & 0.03 \\
\hline & $\mathbf{H}$ & & & & & & & & - \\
\hline \multirow{8}{*}{$\begin{array}{l}\text { Sample } \\
2- \\
\text { Lotion }\end{array}$} & $\mathbf{V}$ & - & 0.39 & 0.00 & -0.05 & 0.29 & 0.69 & 0.29 & 0.40 \\
\hline & $\mathrm{S}$ & & - & 0.04 & 0.05 & 0.20 & 0.37 & 0.02 & -0.06 \\
\hline & $\mathbf{P}$ & & & - & -0.11 & 0.14 & 0.97 & -0.58 & 0.55 \\
\hline & $\mathbf{A}$ & & & & - & -0.15 & 0.52 & 0.47 & 0.27 \\
\hline & $\mathbf{F}$ & & & & & - & 0.47 & 0.67 & 0.39 \\
\hline & $\mathbf{R}$ & & & & & & - & -0.16 & -0.85 \\
\hline & $G$ & & & & & & & - & 0.30 \\
\hline & $\mathbf{H}$ & & & & & & & & - \\
\hline
\end{tabular}

Normalized composite indices were calculated as a product of previous normalized indices, as it is shown in Table 6. Here, "ease of absorption" (0.29) and "residues" (0.34) are the most important attributes under this metric, in the case of Sample 1 and Sample 2, respectively. "Residues" and "freshness" have consistently one of the highest composites indices (0.20 and 0.25 , for sample 1 and 0.34 and 0.14 for sample 2, respectively). In the next section, it is shown how these indices can be used to guide the selection of alternatives, based on the specific profile of each sample product.

Table 6. Normalized values of Shapley (relative weights), Murofushi, and Soneda (interactions) and composite indices. Symbols according to Table 4.

\begin{tabular}{llllllllll}
\hline & & $\mathbf{V}$ & $\mathbf{S}$ & $\mathbf{P}$ & $\mathbf{A}$ & $\mathbf{F}$ & $\mathbf{R}$ & $\mathbf{G}$ & $\mathbf{H}$ \\
\hline \multirow{2}{*}{ Sample 1 Cream } & Normalized relative weights & 0.13 & 0.04 & 0.15 & 0.20 & 0.21 & 0.15 & 0.05 & 0.07 \\
& Normalized interactions & 0.08 & 0.07 & 0.11 & 0.22 & 0.18 & 0.20 & 0.06 & 0.08 \\
& Composite index & 0.07 & 0.02 & 0.11 & $\mathbf{0 . 2 9}$ & $\mathbf{0 . 2 5}$ & $\mathbf{0 . 2 0}$ & 0.02 & 0.04 \\
Sample 2 Lotion & Normalized relative weights & 0.17 & 0.04 & 0.11 & 0.12 & 0.16 & 0.22 & 0.10 & 0.09 \\
& Normalized interactions & 0.11 & 0.06 & 0.13 & 0.09 & 0.12 & 0.21 & 0.13 & 0.15
\end{tabular}




\begin{tabular}{lllllllll}
\hline & V & S & P & A & F & R & G & H \\
\hline Composite index & $\mathbf{0 . 1 4}$ & 0.02 & $\mathbf{0 . 1 0}$ & 0.07 & $\mathbf{0 . 1 4}$ & $\mathbf{0 . 3 4}$ & $\mathbf{0 . 1 0}$ & 0.09 \\
\hline
\end{tabular}

Phase II: Product realization and heuristics update

Starting from a generic basic skin moisturizing cream, identified as K0-N1, which solves the problem specified in Table 2 at a minimum cost, specific changes were made to comply with different attributes for the two evaluated references (Samples 1 and 2). For this, additional heuristics from Table 3 were included in the algorithm as restrictions that allowed searching for feasible alternatives, which increased or reduced the level of the aimed attribute. The resulting formulations are presented in Table E-1 of the Supplementary material.

From the results obtained in the consumer assessment phase for Sample 1, ease of absorption, freshness, and residues were the most important attributes by the composite index. Due to the high interaction between these attributes (see Table 5), and the fact that the base formulation left more white residues than the Sample 1 (Figure 3), the first change made to the algorithm was to implement one of the actions to reduce the white residues by adding up to $2 \%$ low HLB emulsifier (see Table 3). A series of experiments were performed to understand how each change affected the emulsion properties, especially the white residues after product application on skin and the product viscosity. Each one of these alternatives was generated using the optimization problem specified in Table 2. The name of the formulations was given according to the following parameters: The first letter indicates if the product is a cream $(\mathrm{K})$ or a lotion $(\mathrm{L})$, while the number corresponds either to the base case (0) or to the samples 1 or 2 . The following parts also contain letters and numbers. The letter corresponds to the aimed attribute(s) - R for "Residues", G for "Greasiness/oiliness", $\mathrm{V}$ for "Thickness" and $\mathrm{N}$ if no attribute is aimed. The numbers correspond to a counter of the alternatives at this stage.

- Formulation K1-R1 is essentially K0-N1 + 1\% Glyceryl Stearate (replaces water). This could be considered a classical corrective action and is only chosen here to compare with a more systematic approach to solve the problem.

- Formulation K1-R2 includes 1\% Glyceryl Stearate but compensates with the addition of other emollients to keep the minimal surfactant/oil ratio (1:4). The restriction HLB=RLHB is imposed as before in (5).

- Formulation K1-R3 includes 1\% of a low HLB surfactant with the restriction in (6).

- Formulation K1-G1 is made ignoring the importance of indices attributes of the previous section and only taking into account the high value given to the greasiness. This could be considered as an approach to generate a less greasy alternative formulation, based on the consumer assessment, but disregarding the importance and interactions of the attributes. To have a less greasy alternative, the maximal value of $\gamma$ was allowed to be 2.2 instead of 2.4, and K0-N1 left considerably more residues than Sample 2, as can be observed in Figure 3.

For the case of Sample 2, the starting point was set to be Formulation K1-R3, as in this case residues are the most important attribute too (0.34 in the composite index; see Table 6), and from the previous example, it was known that K0-N1 left considerably more residues than Sample 2, as it can be observed in Figure 3. The next action targeted the thickness, as there was no reliable action to correct and easily evaluate the change of the freshness attribute. Two different actions were taken, to observe the results of each action.

- Formulation K2-R3-V1, which comes from the choice of the surfactants, preferring those having oleyl (Polysorbate 80) instead of stearyl groups (Polysorbate 60), as mentioned in Table 3.

- Formulation L2-R3-V1, which considers the same changes and the viscosity parameters of lotions too.

Alternative emulsions characterization

White residues: A visual comparison of the white residues left after application on the skin of the different formulations and the commercial samples are presented in Figure 3. 
As shown here, the white residues of K0-N1 are much higher than that of Sample 1, while for the formulation K1-R1 is very similar to the residues of sample 1, proving that the heuristic about adding a small amount of a low HLB surfactant would reduce the micro-foam generation. This was also the case for sample K1-R3 but not for K1-R2, which did not allow HLB $<$ RHLB. Although K1-G1 was conceived to reduce the greasiness of the base formulation (K0-N1), it also performed better in the white residues. The use of different quantities of emollients also has an impact on the residues left by the product, as it has been said before.
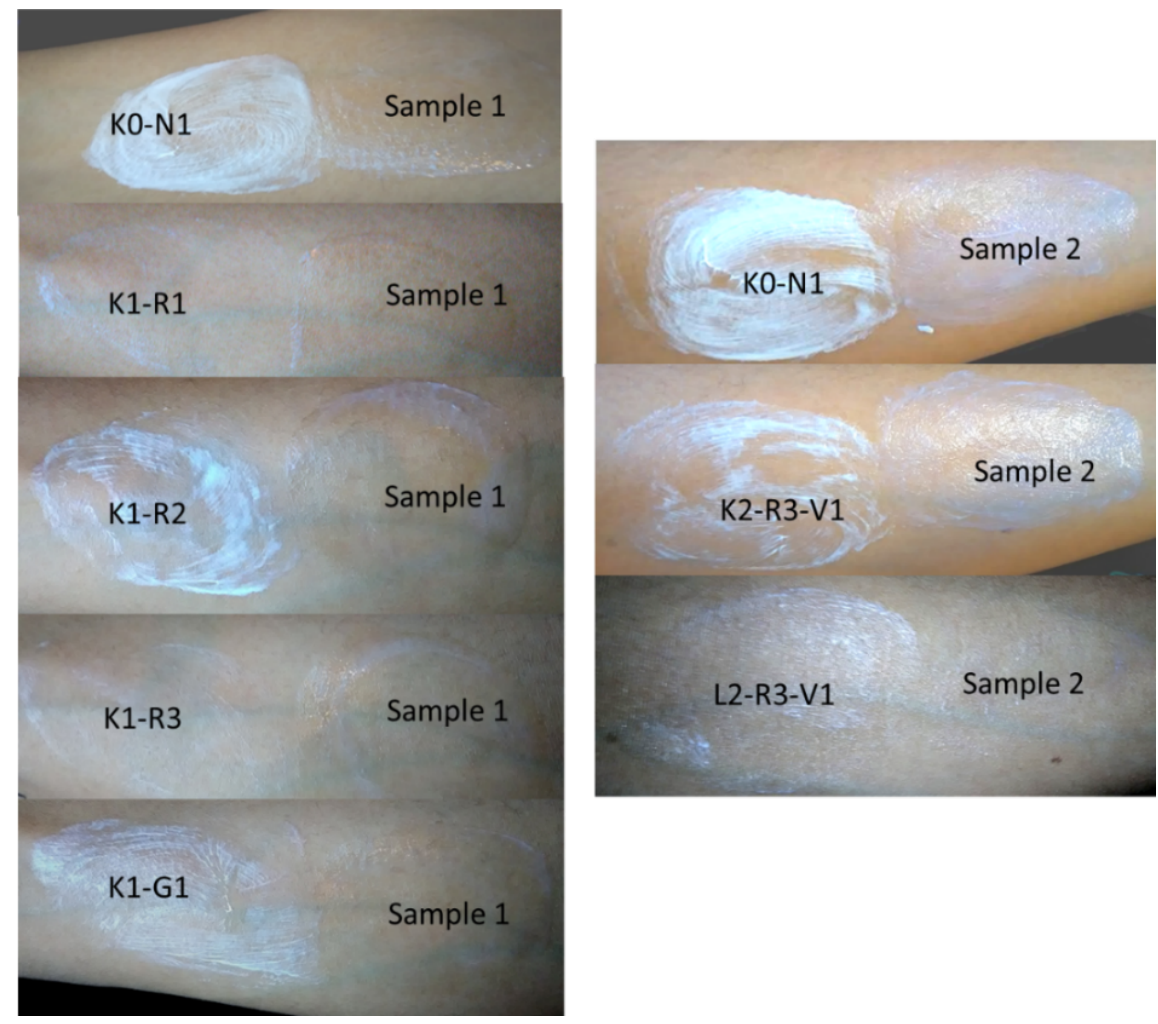

Figure 3: White residues of alternative emulsions compared to Samples 1 (left) and 2 (right)

In the case of Sample 2, the formulation L2-R3-V1, which had into account the residues and the two actions regarding the viscosity of the lotion (restrained values for final viscosity and emulsifiers with oleyl groups), had the best results when compared to the similar alternative K2-R3-V1, which only considered the change of the emulsifiers.

Microscopy: Representative optical microphotographs of Sample 1 and 2 and the prototyped emulsions are presented in Figure 4. It was observed that the base case (K0-N1) had a very different microstructure when compared to Sample 1, with droplet sizes around $5 \mu \mathrm{m}$. It should be noted that the most similar microstructure to sample 1 is the one of formulation K1-R3. In the case of sample 2, the base case (K1-R3) had a very different microstructure when compared to Sample 2, but the formulation L2-R3-V1 is much more similar. 


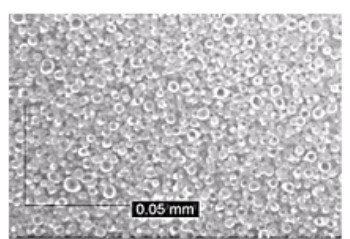

Formulation KO-N1

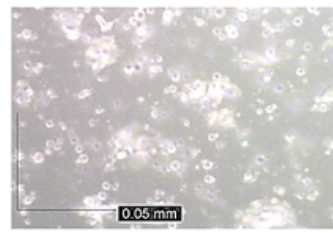

Formulation K1-R3

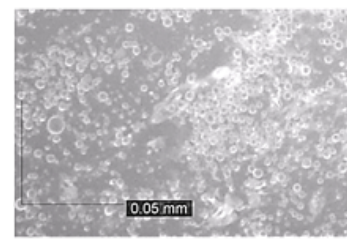

Formulation K2-R3V1

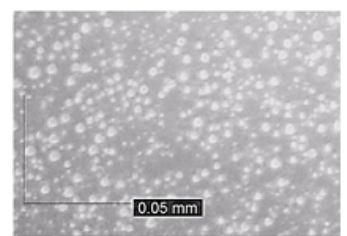

Formulation K1-R1

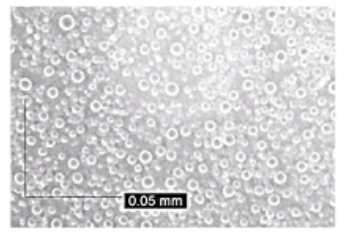

Formulation K1-G1

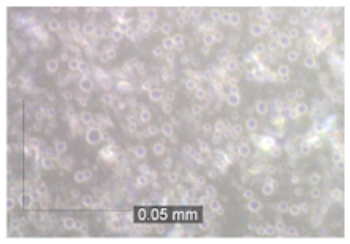

Formulation L2-R3V1

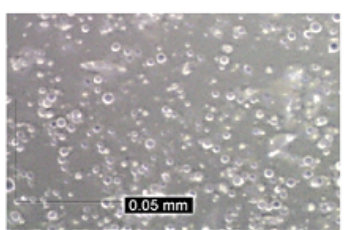

Formulation K1-R2

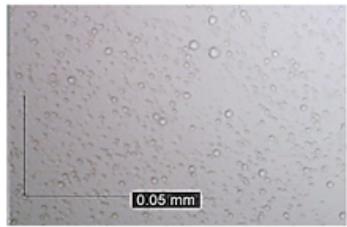

Sample 1

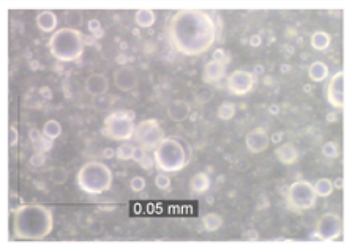

Sample 2

Figure 4: Microphotographs (900X) of the alternative emulsions and the samples 1 and 2

Rheology and textural analysis: The rheological profile of the alternative emulsions is presented in Figure 5. All alternatives have a similar behavior compared to Sample 1. In the case of Sample 2, both formulations L2-R3-V1 and K2-R3-V1 have closer values than K1-R3. Using the power fitting in the experimental data (also shown in Figure 5), it is possible to see that the final viscosities $\eta 2$, calculated for $5000 \mathrm{~s}^{-1}$, lays between 0.023 and $0.5 \mathrm{~Pa} \cdot \mathrm{s}$, for all alternative emulsions. Regarding $\eta 1$, only for Formulation L2-R3-V1 and Sample 2 is possible to observe a plateau and see that this value lays between the ideal limits for lotions (120 and $500 \mathrm{~Pa} \cdot \mathrm{s}$ ), which confirms the suitability of the model to predict emulsion viscosity. 


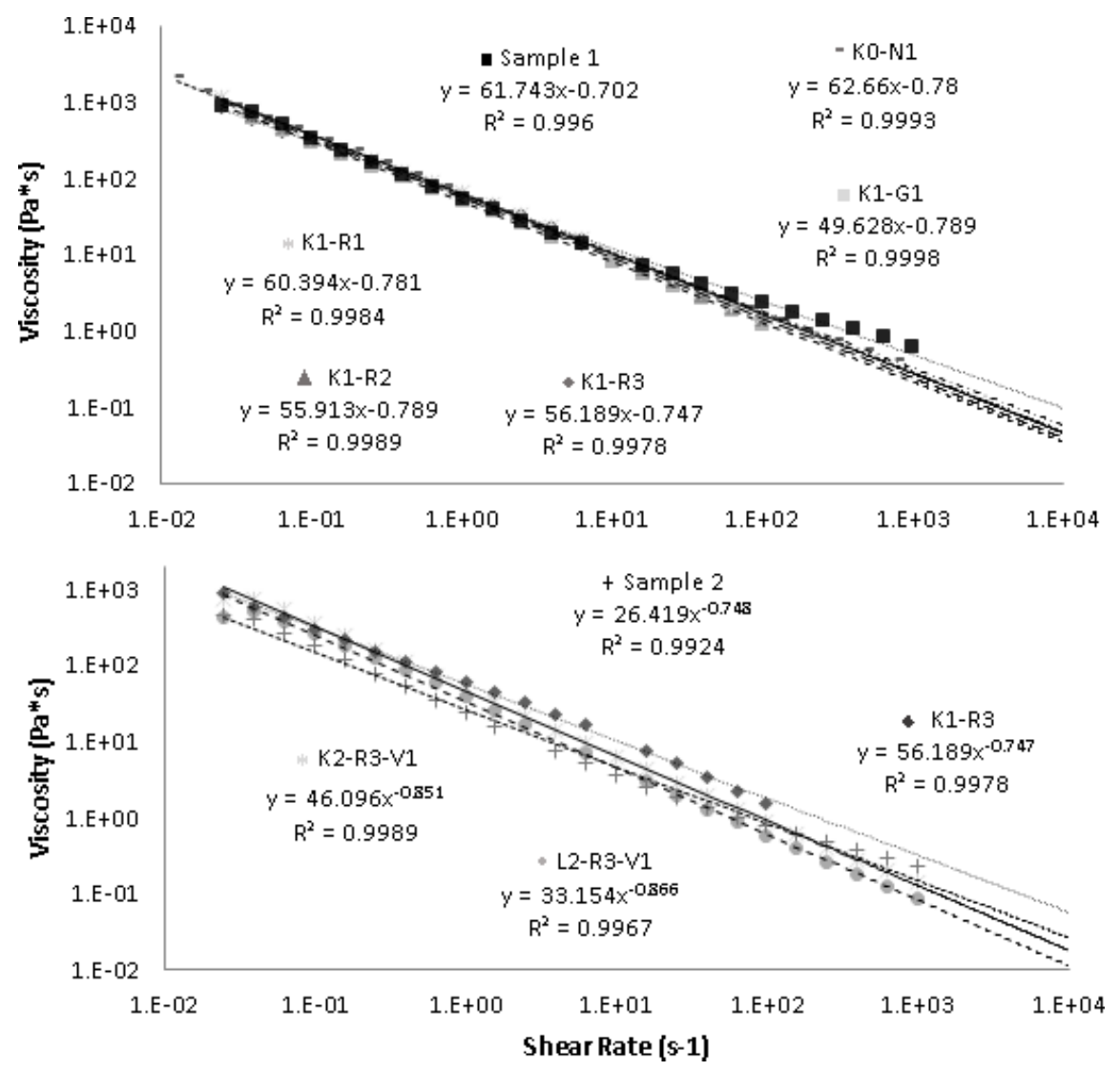

Figure 5: Rheological profile of the alternative emulsions and samples 1 (top) and 2 (bottom)

The parameters obtained from the textural analysis in all prepared sample emulsions are presented in Table 9. The alternative that comes closer to the textural parameters of Sample 1 is K1-R3. For Sample 2, both formulations (L2-R3-V1 and K2-R3-V1) have similar values when compared to Sample 2. Although no sensorial tests were performed on these formulations, it has been reported that the textural parameters here evaluated do correlate well with the sensorial attributes of the final product. In particular, firmness is related to the tackiness as perceived by the user, and consistency, cohesiveness, and index of viscosity all directly correlate with slipperiness. ${ }^{48}$

Table 9. Textural parameters of the alternative emulsions compared to Samples 1 and 2.

\begin{tabular}{lllllll}
\hline & K0-N1 & K1-R1 & K1-R2 & K1-R3 & K1-G1 & Sample 1 \\
Consistency (g.s) & 3979.7 & 6711.4 & 5078.6 & 2131.4 & 6228.0 & 2372.7 \\
Viscosity Index (g.s) & 445.4 & 694.1 & 536.7 & 246.5 & 623.6 & 281.2 \\
Firmness (g) & 154.6 & 285.7 & 199.2 & 81.3 & 244.3 & 90.5 \\
Cohesiveness(g) & 199.1 & 403.0 & 245.2 & 105.8 & 289.0 & 123.5 \\
\hline
\end{tabular}

\begin{tabular}{lllll}
\hline & K1-R3 & L2-R3-V1 & K2-R3-V1 & Sample 2 \\
Consistency (g.s) & 2131.4 & 1929.3 & 2020.6 & 1355.8 \\
Viscosity Index (g.s) & 246.5 & 204.7 & 227.9 & 135.1 \\
Firmness (g) & 81.3 & 73.3 & 78.7 & 52.9 \\
Cohesiveness(g) & 105.8 & 89.6 & 98.6 & 55.1 \\
\hline
\end{tabular}




\section{Conclusions}

In a highly competitive market, end-user involvement has become an important factor in the product design process, as the sensory interactions heavily affect their perception and the assessment of the product. We have proposed an optimization-based methodology for the design of formulated products, which incorporates the assessment of consumer preferences along with available property models and heuristic rules to generate alternative formulations. The methodology was illustrated with two skin moisturizer products and using a commercial product as a reference for each case. A series of alternative solutions with minimum ingredient costs were generated and then manufactured and evaluated. The alternatives generated taking into account the full set of information, including the heuristics update from the consumer assessment data, were shown to be more similar to the reference products. The comparison was made through optical microscopy observations, evaluation of rheological and textural properties, and the measurement of the white residues left during rub-out.

As this methodology involves the participation of untrained consumers, it is possible to reduce the time and resources spent in cosmetic emulsions design compared with sensorial panels, as there is no panel training involved. The methodology permits us to interpret the answer to the products according to how they are perceived and calculate the importance and the interaction of the evaluated attributes, which makes it valuable to the formulator. This suggests that this is a useful methodology not only to formalize the information about consumers' perception of cosmetic products but also to guide the generation of alternatives for other formulated products.

\section{Acknowledgments}

Authors would like to thank BASF, Croda, Evonik, and Provital Group for kindly providing the ingredient samples required to the sample preparation. In addition, authors would like to recognize the involvement of the GEMICO team from the Université de Lorraine for the enabled instruments and guidance for the preparation and characterization of the emulsions. This work was finacially supported by RIFRUTBIO, Convocatoria 617 Colciencias, and by the Universidad Nacional de Colombia within the framework of the research project QUIPU 202010026994. Their support is gratefully acknowledged.

\section{References}

1. Pensé-Lhéritier A-M, ed. Conception Des Produits Cosmétiques : La Formulation. 1st ed. Paris: Lavoisier Tec\&Doc; 2016.

2. Aoussat A, Christofol H, Le Coq M. The new product design - a transverse approach. J Eng Des. 2000;11(4):399-417. doi:10.1080/09544820010000971

3. Rousseau C. Formulation of Cosmetic Products. In: Pense-Lheritier A-M, ed. Formulation. 1st ed. Hoboken, NJ, USA: John Wiley \& Sons, Inc.; 2011:253-268.

4. Parente ME, Gambaro A, Ares G. Sensory characterization of emollients. J Sens Stud. 2008;23(2):149-161. doi:10.1111/j.1745-459X.2007.00136.x

5. Costa R, Moggridge GD, Saraiva PM. Chemical product engineering: An emerging paradigm within chemical engineering. AIChE J. 2006;52(6):1976-1986. doi:10.1002/aic.10880

6. Rahse W. Design of Skin Care Products. In: Brockel PD-IU, Meier DW, Wagner D-IG, eds. Product Design and Engineering: Formulation of Gels and Pastes. Weinheim, Germany: Wiley-VCH Verlag GmbH \& Co. KGaA; 2013:273-313. doi:10.1002/9783527654741.ch10

7. Wibowo C, Ng KM. Product-oriented process synthesis and development: Creams and pastes. AIChE J. 2001;47(12):2746-2767. doi:10.1002/aic.690471214

8. Bagajewicz MJ, Hill S, Robben A, et al. Product design in price-competitive markets: A case study of a skin moisturizing lotion. AIChE J. 2011;57(1):160-177. doi:10.1002/aic.12242 
9. Bernardo FP. Integrated Process and Product Design Optimization. MR, Chemmangattuvalappil NG, eds. Tools for Chemical Product Design: ucts to Biomedicine. Vol 39. Computer Aided Chemical Engineering. In: Martin M, Eden From Consumer Prodhttp://linkinghub.elsevier.com/retrieve/pii/B9780444627001000012.

10. Bernardo FP, Saraiva PM. Integrated process and product design optimization: a cosmetic emulsion application. In: Puigjaner L, Espuna A, eds. Computer Aided Chemical Engineering. Vol 20. Elsevier B.V.; 2005:1507-1512. doi:10.1016/S1570-7946(05)80093-8

11. Cheng YS, Lam KW, Ng KM, Ko RKM, Wibowo C. An integrative approach to product development-A skin-care cream. Comput Chem Eng. 2009;33(5):1097-1113. doi:10.1016/j.compchemeng.2008.10.010

12. Lee CKH, Choy KL, Chan YN. A knowledge-based ingredient formulation system for chemical product development in the personal care industry. Comput Chem Eng. 2014;65:40-53. doi:10.1016/j.compchemeng.2014.03.004

13. Mattei M, Kontogeorgis GM, Gani R. A Systematic Methodology for Design of Emulsion Based Chemical Products. In: Karimi I, Srinivasan R, eds. Computer Aided Chemical Engineering. Vol 31. Elsevier B.V.; 2012:220-224. doi:10.1016/B978-0-444-59507-2.50036-6

14. Pense-Lheritier. Recent developments in the sensorial assessment of cosmetic products: a review. Int J Cosmet Sci. 2015;37(5):n/a-n/a. doi:10.1111/ics.12223

15. Wortel V a. L, Wiechers JW. Skin sensory performance of individual personal care ingredients and marketed personal care products. Food Qual Prefer. 2000;11(1-2):121-127. doi:10.1016/S0950-3293(99)00057-9

16. Civille GV, Dus CA. Evaluating Tactile Properties of Skincare Products: A Descriptive Analysis Technique. Cosmet Toilet. 1991;106(5):83-88.

17. Filipovic M, Lukic M, Djordjevic S, et al. Towards satisfying performance of an $\mathrm{O} / \mathrm{W}$ cosmetic emulsion: screening of reformulation factors on textural and rheological properties using general experimental design. Int J Cosmet Sci. 2017;39(5):486-499. doi:10.1111/ics.12402

18. Lukic M, Jaksic I, Krstonosic V, Dokic L, Savic S. Effect of Small Change in Oil Phase Composition on Rheological and Textural Properties of w/o Emulsion. J Texture Stud. 2013;44(1):34-44. doi:10.1111/j.17454603.2012.00363.x

19. Gilbert L, Picard C, Savary G, Grisel M. Rheological and textural characterization of cosmetic emulsions containing natural and synthetic polymers: Relationships between both data. Colloids Surfaces A Physicochem Eng Asp. 2013;421:150-163. doi:10.1016/j.colsurfa.2013.01.003

20. SRL. Emotional Research. http://www.srlresearch.com/emotional-research/. Published 2018. Accessed June 25, 2018.

21. Lokman AM, Kamaruddin KA. Kansei affinity cluster for affective product design. Proc - 2010 Int Conf User Sci Eng i-USEr 2010. 2010:38-43. doi:10.1109/IUSER.2010.5716719

22. Nagamachi M, Lokman AM. Innovations of Kansei Engineering.; 2011. doi:10.1017/CBO9781107415324.004

23. Nagamachi M. Kansei Engineering: A new ergonomic consumer-oriented technology for product development. Int J Ind Ergon. 1995;15:3-11. doi:10.1016/0169-8141(94)00052-5

24. Yang CC. A classification-based Kansei engineering system for modeling consumers' affective responses and analyzing product form features. Expert Syst Appl. 2011;38(9):11382-11393. doi:10.1016/j.eswa.2011.03.008

25. Shieh M-D, Li Y, Yang C-C. Product Form Design Model Based on Multiobjective Optimization and Multicriteria Decision-Making. Math Probl Eng. 2017;2017. doi:10.1155/2017/5187521 
26. Keeney RL, Raiffa H. Decisions with Multiple Objectives - Preferences and Value Tradeoffs. Cambridge: Cambridge University Press; 1993.

27. Tanaka K, Sugeno M. A study on subjective evaluations of printed color images. Int J Approx Reason. 1991;5(3):213-222. doi:10.1016/0888-613X(91)90009-B

28. Schmitt E, Bombardier V, Wendling L. Improving fuzzy rule classifier by extracting suitable features from capacities with respect to the Choquet integral. IEEE Trans Syst Man, Cybern Part B Cybern. 2008;38(5):1195-1206. doi:10.1109/TSMCB.2008.925750

29. Camargo M, Wendling L, Bonjour E. A fuzzy integral based methodology to elicit semantic spaces in usability tests. Int J Ind Ergon. 2014;44(1):11-17. doi:10.1016/j.ergon.2013.08.007

30. Arrieta-Escobar JA, Bernardo FP, Orjuela A, Camargo M, Morel L. Incorporation of heuristic knowledge in the optimal design of formulated products: Application to a cosmetic emulsion. Comput Chem Eng. 2019;122:265-274. doi:10.1016/j.compchemeng.2018.08.032

31. Arrieta-Escobar JA, Bernardo FP, Orjuela A, Camargo M, Morel L. An Integrated Methodology for Emulsified Cosmetic Product Formulation Using Integer Programming with Logical Constraints. In: Espuna A, Graells M, Puigjaner L, eds. Computer Aided Chemical Engineering. Vol 40. Barcelona, Spain: Elsevier B.V.; 2017:985-990. doi:10.1016/B978-0-444-63965-3.50166-5

32. Arrieta-Escobar JA, Bernardo FP, Orjuela A, Camargo M, Morel L, Wendling L. Integration of Consumer Preferences and Heuristic Knowledge in the Design of Formulated Products: Application to a Cosmetic Emulsion. In: Kiss AA, Zondervan E, Lakerveld R, Ozkan L, eds. 29th European Symposium on Computer Aided Process Engineering. Vol 46. Eindhoven: Elsevier B.V; 2019:433-438. doi:10.1016/B978-0-12-8186343.50073-4

33. Grabisch M. Fuzzy integral in multicriteria decision making. Fuzzy Sets Syst. 1995;69:279-298. doi:10.1016/0165-0114(94)00174-6

34. Grabisch M. A New Algorithm for Identifying Fuzzy Measures and Its Application to Pattern Recognition. Int Jt Conf Fourth IEEE Int Conf Fuzzy Syst Second Int Fuzzy Eng Symp. 1995;1(ii):145-150. doi:http://dx.doi.org/10.1109/FUZZY.1995.409673

35. Choquet N. Theory of capacities. Ann l'Institut Fourier. 1954;5:131-295.

36. Grabisch M. The application of fuzzy integrals in multicriteria decision making. Eur J Oper Res. 1996;89(3):445-456. doi:Doi 10.1016/0377-2217(95)00176-X

37. Mattei M. Advances in Chemical Product Design - An Integrated Methodology for Emulsified Formulated Product Design. 2014;(July).

38. Parente ME, Manzoni AV, Ares G. External Preference Mapping Of Commercial Antiaging Creams Based On Consumers' Responses To A Check-All-That-Apply Question. J Sens Stud. 2011;26(2):158-166. doi:10.1111/j.1745-459X.2011.00332.x

39. Osgood CE. Semantic Space Revisited. WORD. 1959;15(1):192-200. doi:10.1080/00437956.1959.11659693

40. Shapley LS. QUOTA SOLUTIONS OF n-PERSON GAMES1. Contrib to Theory Games, Vol II(AM-28). 1953;2:343.

41. Murofushi T, Sugeno M. A theory of fuzzy measures: Representations, the Choquet integral, and null sets. J Math Anal Appl. 1991;159(2):532-549. doi:10.1016/0022-247X(91)90213-J

42. Bernardo FP, Saraiva PM. A conceptual model for chemical product design. AIChE J. 2015;61(3):802815. doi:10.1002/aic.14681 
43. Eccleston GM. Functions of mixed emulsifiers and emulsifying waxes in dermatological lotions and creams. Colloids Surfaces A Physicochem Eng Asp. 1997;123-124:169-182. doi:10.1016/S0927$7757(96) 03846-0$

44. O'Lenick T. Exclusive ! Comparatively Speaking : Cream vs . Lotion. Cosmetics \& Toiletries. http://www.cosmeticsandtoiletries.com/research/chemistry/3268716.htm. Published 2008. Accessed September 4, 2015.

45. Kostansek E. Emulsions. In: Kirk-Othmer Chemical Technology of Cosmetics. Hoboken, NJ, USA: John Wiley \& Sons, Inc.; 2012:551-574. http://books.google.com/books?id=nxO7xkQ0wFsC\&pgis=1.

46. Barel AO, Paye M, Maibach HI. Handbook of Cosmetic Science and Technology. New York - Basel: Marcel Dekker; 2001.

47. Korhonen M, Niskanen H, Kiesvaara J, Yliruusi J. Determination of optimal combination of surfactants in creams using rheology measurements. Int J Pharm. 2000;197:143-151.

48. Lukic M, Jaksic I, Krstonosic V, Cekic N, Savic S. A combined approach in characterization of an effective w/o hand cream: The influence of emollient on textural, sensorial and in vivo skin performance. Int J Cosmet Sci. 2012;34(2):140-149. doi:10.1111/j.1468-2494.2011.00693.x

49. Moravkova T, Filip P. Relation between sensory analysis and rheology of body lotions. Int J Cosmet Sci. 2016;38(6):558-566. doi:10.1111/ics.12319

50. Dubuisson P, Picard C, Grisel M, Savary G. How does composition influence the texture of cosmetic emulsions? Colloids Surfaces A Physicochem Eng Asp. 2018;536(July 2016):38-46. doi:10.1016/j.colsurfa.2017.08.001

51. Munoz J, Alfaro C. Avances en la formulacion de emulsiones. Grasas Y Aceites. 2007;3495(1):64-73.

52. Wiechers JW, Kelly CL, Blease TG, Dederen JC. Formulating for efficacy. Int J Cosmet Sci. 2004;26:173182. doi:10.1111/j.1467-2494.2004.00211.x

53. Moravkova T, Filip P. The Influence of Emulsifier on Rheological and Sensory Properties of Cosmetic Lotions. Adv Mater Sci Eng. 2013;2013(6):1-7. doi:10.1155/2013/168503

54. Brummer R, Godersky S. Rheological studies to objectify sensations occurring when cosmetic emulsions are applied to the skin. Colloids Surfaces A Physicochem Eng Asp. 1999;152(1-2):89-94. doi:10.1016/S0927$7757(98) 00626-8$

55. Parente ME, Ares G, Manzoni AV. Application of two consumer profiling techniques to cosmetic emulsions. J Sens Stud. 2010;25(5):685-705. doi:10.1111/j.1745-459X.2010.00297.x

56. Ansmann A, Busch P, Hensen H, Hill K, Krachter H-U, Muller M. Personal Care Formulations. In: Showell M, ed. Handbook of Detergents, Part D: Formulation. Surfactant Science. CRC Press; 2005:207260. doi:10.1201/9781420028713.ch7

57. Dederen JC, Chavan B, Rawlings A V. Emollients are more than sensory ingredients: The case of Isostearyl Isostearate. Int J Cosmet Sci. 2012;34(6):502-510. doi:10.1111/j.1468-2494.2012.00744.x

58. Mentel M, Wiechers S, Howe A, Biehl P, Meyer J. Senses- A Scientific Tool for the Selection of The Right Emollient. SOFW. 2014;140(8):8-15.

59. Rieger MM, Rhein LD, eds. Surfactants in Cosmetics. Vol 68. 2nd ed. New York: Marcel Dekker; 1997.

60. Iwata H, Shimada K. Formulas, Ingredients and Production of Cosmetics. Tokyo: Springer; 2013. doi:10.1007/978-4-431-54061-8

61. Wang S, Kislalioglu MS, Breuer M. The Effect of Rheological Properties of Experimental Moisturizing Creams/Lotions on Their Efficacy and Perceptual Attributes. Int J Cosmet Sci. 1999;21(3):167-188. 
doi:10.1046/j.1467-2494.1999.203162.x

62. Prospector(R). UL Prospector. https://www.ulprospector.com/en/la/PersonalCare. Published 2017. Accessed September 13, 2017.

63. Pal R. Evaluation of theoretical viscosity models for concentrated emulsions at low capillary numbers. Chem Eng J. 2001;81(1-3):15-21. doi:10.1016/S1385-8947(00)00174-1

64. ASTM E1490-03. Standard Practice for Descriptive Skinfeel Analysis of Creams and Lotions. West Conshohocken, PA,, PA,: ASTM International; 2003. doi:10.1520/E1490-03

65. Institute of Personal Care Science. Free formulas/Videos. https://personalcarescience.com.au/Freeformulas-Videos/FreeVideos-448/. Published 2016. Accessed March 7, 2018.

66. Sakamoto K, Lochhead RY, Maibach HI, Yamashita Y, eds. Cosmetic Science and Technology: Theoretical Principles and Applications. 1st ed. Amsterdam, Oxford, Cambridge: Elsevier; 2017. 